\title{
Applicability evaluation of the stress-optic law in Newtonian fluids toward stress field measurements
}

\author{
Daisuke Noto $\mathbb{Q}^{*}$ ' Yuji Tasaka $\odot$, Jumpei Hitomi, and Yuichi Murai $\odot$ \\ Laboratory for Flow Control, Faculty of Engineering, Hokkaido University, N13W8, Sapporo 060-8628, Japan
}

(Received 6 May 2020; accepted 4 October 2020; published 21 October 2020)

\begin{abstract}
A novel rheoscopic fluid displaying coloration due to flow-induced birefringence is investigated through laboratory experiments to ascertain if it satisfies the stress-optic law in fluids in direct stress field measurements of Newtonian fluids. To validate this experimentally, two types of spin-up experiments ensuring two-dimensional flows were performed: color visualization using the rheoscopic fluid and velocity field measurement by particle image velocimetry. Using a wave-number analysis, the orientation angle of the birefringence was determined from a single color snapshot in axisymmetric and unidirectional flows. Direct comparisons between color and shear stress values made it possible to establish a unique relation between the two, verifying that the stress-optic law can be applied in the fluid. The results of the present study suggest a promising avenue toward enabling temporally and spatially resolved stress field measurements in Newtonian fluids.
\end{abstract}

DOI: 10.1103/PhysRevResearch.2.043111

\section{INTRODUCTION}

Direct measurements of stress fields in fluid flows are useful to understand details of fluid physics and mechanics. For Newtonian fluids, such as water and air, the stress tensor $\boldsymbol{\tau}$ is linearly proportional to the strain rate $\nabla \mathbf{u}$ with a constant viscosity $\mu$ as $\boldsymbol{\tau}=\mu \nabla \mathbf{u}$. However, measurements of the fields of the spatially resolved velocity gradient tensor $\nabla \mathbf{u}$ in fluid flows is not straightforward, because in practical flows, the accessible value is the velocity field, not the gradient of the field.

Particle image velocimetry (PIV) makes it possible to measure flow fields in cross sections of flows with simple planar PIV [1,2]. Then by determining derivatives of the velocity vector fields, physical quantities, including the vorticity, shear rate, and other parameters, can be calculated in postprocessing. The qualities of the derivative values are directly affected by both the dynamic range and the spatial resolution of the velocity vector fields. The velocity vectors are determined by identifying dominant displacements of particles in minute regions of two images recorded at short time intervals, and the resulting velocity vectors are smoothed within the interrogation region, limiting the dynamic range of the PIV. Additionally, the spatial resolution of PIV is typically orders of magnitude poorer than that of the original image and will be too small for the acquisition of the fine details of derivatives of velocity fields. Efforts to measure the derivatives of velocity fields have been made [3], but currently, PIV does not satisfy the desired needs for direct fluid deformation measurements,

\footnotetext{
*noto@ ring-me.eng.hokudai.ac.jp
}

Published by the American Physical Society under the terms of the Creative Commons Attribution 4.0 International license. Further distribution of this work must maintain attribution to the author(s) and the published article's title, journal citation, and DOI. as far as the derivatives cannot be measured as the first observation values.

An alternative to direct measurements of fluid deformation, molecular tagging velocimetry (MTV), is summarized by Tropea and Yarin [4]. In MTV, numerous "tags" are created in fluorescent fluids using a laser beam lattice. Velocity vectors are quantified by tracking the centroids of the tags without needing the seeding with tracer particles. Shear rates can be obtained from the deformation of the shape of the tags without knowing the derivatives of the velocity vectors, even in multiphase turbulent flows $[5,6]$. The established work has focused on turbulent flows and was applied to obtain statistics of the turbulent flows, and the spatial resolution of the shear rate measurements obtained in this manner is not applicable to field measurements as the spatial resolution of MTV depends on the distances between the beams forming the beam lattice and is equivalent to or poorer than PIV.

In different research fields, such as geology and materials science, stress or strain field measurements utilizing the stress-optic law have been conducted for several decades, as reviewed by Patterson [7]. The stress-optic law is written as

$$
\boldsymbol{n}_{i j}=C \boldsymbol{\tau}_{i j},
$$

where $\boldsymbol{n}_{i j}, \boldsymbol{\tau}_{i j}$, and $C$ are the refractive index tensor, the stress tensor, and the photoelastic (stress-optical) coefficient, respectively. The internal stress of a solid material induces birefringence, and a light ray passing through the material is affected by showing changes in coloration. Backlight projection with optical filters enables observations of the internal stress distributions of solid materials like in plastic resins, even with irregular geometries. This technique is termed photoelasticity (photoelastic analysis), and various methods to quantify the birefringence observed here have been developed as summarized by Ramesh et al. [8].

Photoelastic analysis in fluid flows has also been reported [9-11]. These reports investigated the stress-optic law as it is manifested in flows of polymer solutions (i.e., non-Newtonian 


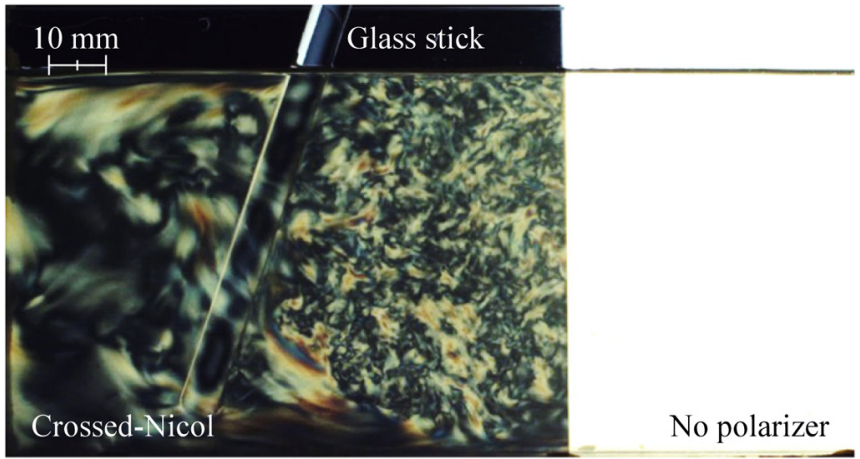

FIG. 1. An example of flow visualization using the rheoscopic fluid with the crossed-Nicol method (left half) and without polarizers (right half). The fluid was disturbed by a moving glass stick.

fluids). The birefringence is induced by fluid deformations in such non-Newtonian fluids by analogy with solid materials and termed a flow-induced birefringence. The anisotropy originating in the polymer structures provides the rheological properties of the fluids, and the stress-optic law in fluids is applied in the field of rheology and known as rheo-optics. For Newtonian fluids, however, it is not directly possible to show the flow-induced birefringence because the fluid is isotropic. Classically, rheoscopic fluids containing small flake particles, such as aluminum flakes and mica, have been used for qualitative visualization of Newtonian fluid flows with coherent flow patterns [12]. The flake particles are considered to preferentially align in the principal shear direction in the flow, even though actual particle motions are much more complicated [13-15]. A recent report seeking an alternative to classical rheoscopic fluids by Borrero-Echeverry et al. [16] introduced the use of shaving cream containing stearic acid to provide anisotropy. Such anisotropic additives are, however, too large to affect light rays.

The flow-induced birefringence was utilized to a Newtonian fluid by $\mathrm{Hu}$ et al. [17,18], where wakes around a swimming fish were visualized by the tobacco mosaic virus (rod-shaped particles). Laminar Sciences Corporation [19] has produced a novel rheoscopic fluid named KaleidoFlow as a birefringent Newtonian fluid composed of tiny flake particles and deionized water (as detailed in Sec. II A). This fluid shows local fluid deformations by displaying multiple colors as shown in Fig. 1; the fluid was poured into a 10-mm-thick rectangular vessel and disturbed by a moving glass stick. In Fig. 1, the left half of the vessel is sandwiched by two orthogonally arranged polarization filters, the so-called crossed-Nicol method, for simultaneous visualizations of backlight without polarizer (the right half) and the crossed-Nicol images. Complicated color patterns generated by the flow-induced birefringence can be seen surrounding the glass stick in the left half, while no flow structures are observed in the right half.

As detailed in $\mathrm{Hu}$ et al. [18], the birefringence in these Newtonian fluids is elicited by the preferential alignments of the anisotropic particles in the shear direction, similar to the classical rheoscopic fluids. The cumulative effect of particle orientation along the optical axis deviates the polarization plane of the light emerging from the polarizer, thus generating
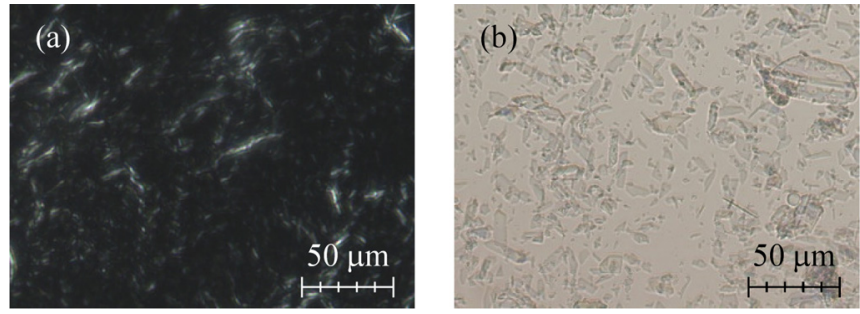

FIG. 2. Microscope photos of two different rheoscopic fluids: (a) the rheoscopic fluid used in this study, KaleidoFlow (Laminar Science Corp. [19]), recorded with a crossed-Nicol method, and (b) the classical rheoscopic fluid, AQ-1000 (Kalliroscope), taken with a opened-Nicol method.

a bright coloration. By increasing the shear rate, the particles become more and more oriented in the shear direction until a saturation corresponding to a plateau of birefringence, a particle orientation perfectly in the shear direction. Hence it is expected that the stress-optic law in the fluids may be valid until certain high values of shear rate, if the stress-optic law is applicable for the photoelasticity of the Newtonian rheoscopic fluid.

The present study aims to investigate if the stress-optic law holds in the Newtonian rheoscopic fluid and to explore a doable way of conducting photoelastic analysis or stress field measurement in experimental investigations. First, the coloration mechanism of the rheoscopic fluid and the applicable flow conditions for photoelastic analysis in fluid flows will be discussed in Sec. II. Second, a detailed investigation of laboratory experiments is conducted under conditions fulfilling the limitations and will be discussed in Sec. III. In Sec. IV, the stress-optic law in the fluid will be evaluated using the experimental results. Finally, a summary of the present study and plausible ways to conduct future stress field measurements in Newtonian fluids will be provided in Sec. V.

\section{PHOTOELASTICITY IN A NEWTONIAN FLUID}

\section{A. Flow visualizer}

Specifications of the novel rheoscopic fluid, KaleidoFlow, are available in patents by Laminar Sciences Corporation $[20,21]$. According to the documents, a small amount of flake- or plate-structured vermiculite particles is dispersed into deionized water. The typical particle dimensions are $O(10 \mu \mathrm{m})$ in longitudinal directions and $O(10 \AA)$ in thickness, and the specific gravity is $\sim 2.65$. The particles are homogeneously dispersed in the deionized solvent, and sedimentation of the particles occurs finally after standing the fluid for a week. For a wide color spectrum coloration, the electrical conductivity of the solvent needs to be very small. In our preliminary test for diluting the rheoscopic fluid, the use of tap water for diluting induced the flocculation of the vermiculite particles. A microscope photo of the rheoscopic fluid taken with a crossed-Nicol method is shown in Fig. 2(a). For comparison, a microscope photo of the classical rheoscopic fluid, Kalliroscope [12], taken with a single linear polarizer (opened-Nicol method), is shown in Fig. 2(b). Flake-structured particles of the Kalliroscope, length scale $O\left(10^{-6}-10^{-5} \mathrm{~m}\right)$, can be observed in Fig. 2(b). Different 


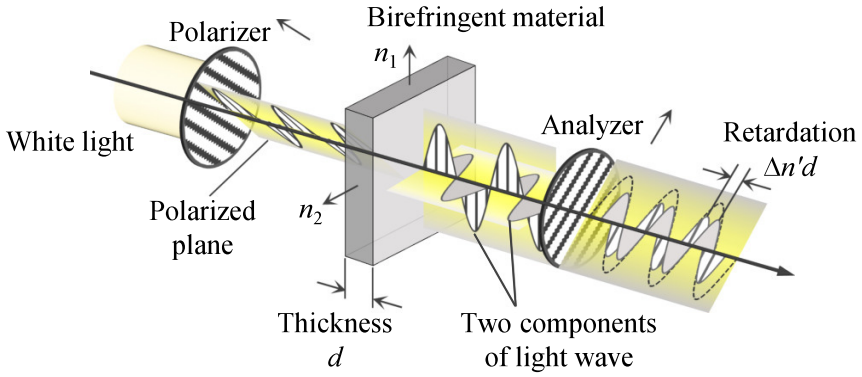

FIG. 3. Schematic illustration of the coloration mechanism caused by a birefringent material.

from this, no clear shapes of the KaleidoFlow are visible at the same magnification in Fig. 2(a). These photos suggest that the size of the particles in the KaleidoFlow are much smaller than those of the Kalliroscope, and it is concluded that the novel rheoscopic fluid affects visible light rays directly by optical anisotropy like liquid crystals and displays coloration due to flow-induced birefringence, as shown in Fig. 1, despite the medium being a Newtonian fluid. As it was not possible to recognize flake particles in the diluted rheoscopic fluid with naked eyes, the expected spatial resolution of the visualization can be deduced to be similar to or better than $O(10 \mu \mathrm{m})$. This fine spatial resolution is much better than the general PIV technique requiring seeding tracer particles of $O(10-100 \mu \mathrm{m})$ in diameter.

\section{B. Mechanism of coloration}

As the mechanism of the flow-induced birefringence utilizing anisotropic particles is detailed in Hu et al. [18], a general coloration mechanism is provided in this section. Coloration like in Fig. 1 emerges when an optically anisotropic (birefringent) material is placed between two orthogonally arranged linear polarizers and lit up from behind by a white light. A schematic illustration of this coloration mechanism simplified into a two-dimensional system is shown in Fig. 3. A white light placed in front of (left in the figure) the first polarization filter in front of the birefringent component with thickness $d$, termed the polarizer, vibrates in all directions perpendicular to the traveling direction of the light. After the polarizer, only the light ray component vibrating in the direction of the polarizer passes through the polarizer, and this polarized light enters the birefringent material. The polarized light is doubly refracted and separated into two components vibrating along the optical axes of the material perpendicular to each other, noted as $n_{1}$ and $n_{2}$ in Fig. 3. Then the polarized light rays pass through the material and enter the second polarization filter, termed the analyzer. Due to the velocity difference between the two light rays passing through the material, the light rays are elliptically polarized, and the analyzer lets only the light ray component parallel to itself pass.

The light intensity $I$ transmitted from the white light source irradiated from the back through these optical settings is theoretically expressed as

$$
I=I_{0} \sin ^{2} 2 \chi \sin ^{2} \frac{\pi \Gamma}{\lambda},
$$

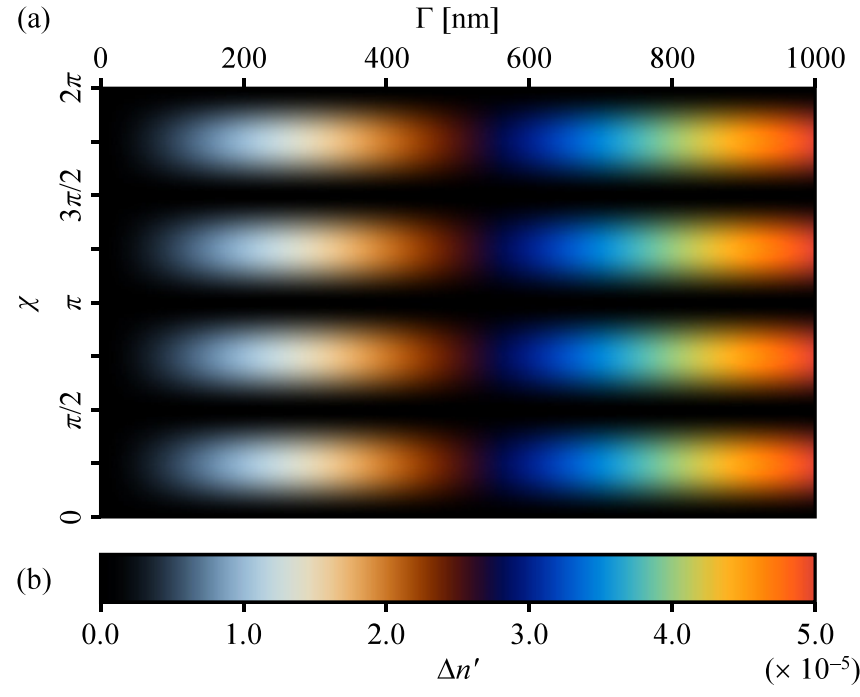

FIG. 4. Michel-Lévy interference color charts obtained in the present system at a fixed $d=20 \mathrm{~mm}$ : (a) a full color chart with both $\Delta n^{\prime}$ and $\chi$ as variables and (b) extracted brightest coloration at $\chi=\pi / 4$.

where $I_{0}, \chi(0 \leqslant \chi \leqslant \pi / 4), \Gamma$, and $\lambda$ represent the original light intensity, the angular difference between the optical axis of the material and the polarizer axes, the so-called orientation angle, the retardation (optical path difference), and the wavelength of the light, respectively. $\pi \Gamma / \lambda$ has been termed the phase difference of the two light rays in some reports. The retardation $\Gamma$ has a length scale of $O\left(10^{-6} \mathrm{~m}\right)$ and is defined as

$$
\Gamma=\left|\Delta n^{\prime}\right| d=\left|n_{1}-n_{2}\right| d,
$$

where $\left|\Delta n^{\prime}\right|$ is the difference between the two refractive indices, the larger one $n_{1}$ and the smaller one $n_{2}$, termed the birefringence of the material, and $d$ denotes the length of the optical path (equivalent to the material thickness) as shown in Fig. 3. Substituting Eq. (3) into Eq. (2), we obtain

$$
I=I_{0} \sin ^{2} 2 \chi \sin ^{2} \frac{\pi \Delta n^{\prime} d}{\lambda} .
$$

Here $\left|\Delta n^{\prime}\right|$ is defined as $\Delta n^{\prime}(\geqslant 0)$ to enhance readability. In practice, $I_{0}$ is the intensity of the original light source and is a specific function of $\lambda$ with a fixed light source. Further, $d$ is a constant at a set material thickness, and thus $\Gamma$ is a scaled value of $\Delta n^{\prime}$. Finally, only two variables, $\chi$ and $\Delta n^{\prime}$, determine the behavior of $I$, displaying a specific color fringe pattern, termed the Michel-Lévy interference color chart.

A detailed development process for the color chart was reported by Sørensen [22]. The color chart was obtained numerically by using the spectrum of the light used in the present study with a fixed material thickness $(d=20 \mathrm{~mm})$ and shown in Fig. 4. As a result of the $I$ transmitted by the birefringent material, the coloration is recorded as RGB brightness values in digital images. Please note that the color chart is a function of both $\Delta n^{\prime}$ (or $\Gamma$ with a set $d$ ) in the horizontal axis and $\chi$ in the vertical axis of Fig. 4(a). With increases in $\Delta n^{\prime}$, the color chart displays an increasingly vivid coloration. Contrary to this, four of the same color cycles appear with variations of $\chi$; 
dark at $\chi=m \pi / 2$ and bright at $(2 m-1) \pi / 4$, with arbitrary integer $m \in[1,4]$. This color cycle is consistent with Eq. (4) as $I \propto \sin ^{2} 2 \chi$. For a detailed view of the brightest coloration, this color at $\chi=\pi / 4$ is extracted in Fig. 4(b), where there are two dark-colored sectors at $\Delta n^{\prime}=0.0$ and $\Delta n^{\prime} \sim 2.8 \times 10^{-5}$. The color chart continues at further larger $\Delta n^{\prime}$ regions, which is not shown in the figure. From the color chart it is clear that both $\chi$ and $\Delta n^{\prime}$ are necessary to determine a unique color in the chart.

\section{Applicable flow conditions}

The most significant outstanding issue is whether the stress-optic law holds in the flow-induced birefringence of the rheoscopic fluid considered here. To determine this, flowinduced birefringence $\Delta n^{\prime}$ and orientation angle $\chi$ must be associated uniquely with the stress in a specific flow by laboratory experiments.

The photoelastic analysis requires backlight illumination, and light rays transmitted through a birefringent material are recorded as the color information. This makes the coloration the result of an integration of the material state over the depth (in the light ray direction). For solid materials, this need not be a significant problem because thin structures are required for the backlight illumination, ensuring that one-dimensional (1D) or two-dimensional (2D) structures are acceptable and that the material state can be regarded as identical over the full depth of the sample. Fluid flows easily form threedimensional (3D) structures, and to evaluate a proper $\Delta n^{\prime}$ using a single optical setting, the flows are required to be confined in thin fluid vessels like in Hele-Shaw cells to ensure the minimum length scale of the flow to be equivalent to or larger than the thickness of the fluid vessel. Otherwise, 1D or 2D fluid flows must be able to be assumed by the presence of external forces such as rotation.

\section{Requirements for fluid flow measurement}

To surpass the limitations of conventional methods such as PIV and MTV, the photoelastic analysis in the fluid flows is required to possess high temporal and spatial resolutions. In the photoelastic analysis, determination of $\chi$, the vertical axis of Fig. 4(a), could present a bottleneck. For solid materials, $\chi$ is easily obtained by rotating one polarizer or the materials, because the transmitted color repeats bright and dark cycles as shown in Fig. 4(a). To ensure a correct determination of $\chi$, at least four images taken with two linear polarizers aligned at different angles are required [23]. This would be efficient because the solid materials do not change the states during the rotation of the polarizer, i.e., solid materials may be regarded as offering a steady state under fixed externally imposed conditions, and no time lag will be required in the determination of $\chi$. A fluid in the rotating vessel, however, will present changes in the flow state during rotation. This makes it necessary for $\chi$ to be quantified instantaneously or within a very short period of time. Without near instantaneous temporal resolution measurement, targets for the photoelastic analysis in fluid flows become limited to steady flows.

As introduced in Sec. I, methods of analysis other than photoelastic stress field measurements are possible with other conventional methods, such as PIV and MTV. The spatial resolutions of these conventional methods, however, are not fully detailed full-field measurements, and in practice they offer ten-times-poorer spatial resolution than that of the image resolution, and details of small-scale structures and the large velocity gradients generated in turbulent flows are not resolved. To get around this limitation in spatial resolution, the use of pixel-wise information could be useful, and photoelastic analysis offers advantages for achieving this and would make it possible to quantify internal stress distributions also in irregularly shaped solid materials.

The present investigation attempts to verify the validity of the stress-optic law in rheoscopic fluid flows by using laboratory experiments to achieve results similar to those for solid materials immediately above.

\section{FLOW VISUALIZATION IN SPIN-UP FLUID FLOW}

\section{A. Spin-up experiment}

As a first step in evaluating the validity of the stress-optic law in 1D or 2D fluid flows, spin-up flow was chosen as the measurement target. Spin-up flows have a long history of investigations (e.g., [24-27]), and here the experiments ensure axisymmetry, and unidirectionality in the azimuthal direction, also during the transient state. Thus the limitations of steady flow measurements can be overcome.

In the present study, two series of spin-up experiments were conducted by confining two different fluids in a small cylindrical vessel with depth $d=20 \mathrm{~mm}$ and inner diameter $D=80 \mathrm{~mm}$. One is a color visualization experiment using the rheoscopic fluid diluted with the same volume of distilled water; the physical properties of the diluted rheoscopic fluid are not different from water, because the fluid is composed of deionized water with a tiny amount of additives. The second series is a velocity field measurement using distilled water containing small amounts of porous resin particles (mean diameter $d_{p}=90 \mu \mathrm{m}$, mean density $\rho_{p}=1.02 \times 10^{3} \mathrm{~kg} / \mathrm{m}^{3}$, HP20SS, Mitsubishi Chemical Co.) as tracers for PIV. Schematic diagrams of the experimental setups are shown in Fig. 5. The image acquisition for both was made with a digital camera (D3400, Nikon) with a lens (Nikkor $35 \mathrm{~mm}$, Nikon) fixed above the fluid vessel and recording color images at 25 f.p.s. with shutter speed of $1 / 500 \mathrm{~s}$, aperture of $\mathrm{f} / 2.8$, and a spatial resolution of 0.1 $\mathrm{mm} /$ pixel. As discussed in Sec. II A, the expected spatial resolution of the flow-induced birefringence is $O(10 \mu \mathrm{m})$, each pixel represents cumulative values within the area, and pixel-wise information can be used in this camera configuration. For the color visualization, the fluid container with the rheoscopic fluid was sandwiched between two orthogonally arranged linear polarizers placed on an LED white backlight source (maximum brightness $21000 \mathrm{~cd} / \mathrm{m}^{2}$, HDSBPA, Hayashi-Repic). Please note that the selection of the white light source does not affect the outline of the analysis procedure and discussions to be presented in this study. Here, the polarization axes of the polarizer and the analyzer are parallel to the $y$ and $x$ axes of the image, respectively. For the velocity measurements, the image acquisition was conducted at the same camera settings by placing the container on a stage, 


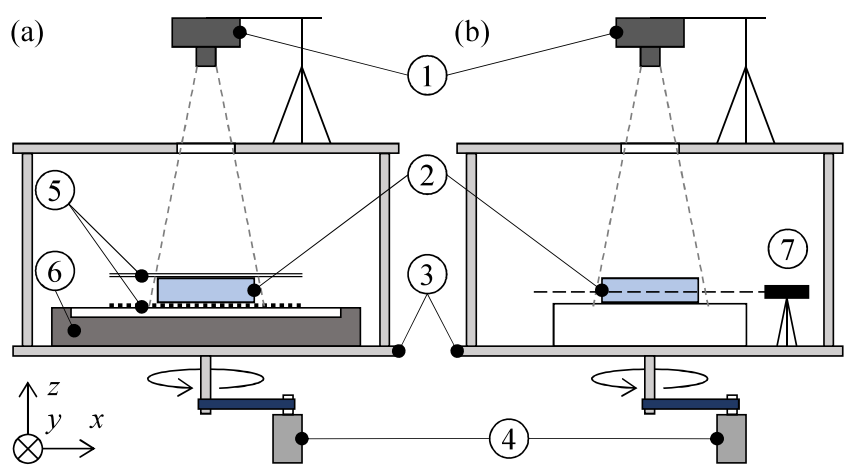

FIG. 5. Schematic diagrams of the experimental setup for (a) color visualization using the rheoscopic fluid and (b) velocity field measurement (PIV) using water: (1) a digital camera, (2) a cylindrical fluid container, (3) turntable with two stages (1 m diameter), and (4) a motor for rotation are used in common for this facility; (5) two orthogonally arranged linear polarization filters and (6) a white backlight source used only for (a) in the color visualization, and (7) a 2-W green laser sheet used only for (b) with the velocity field measurements.

and a horizontal cross section at $z=0.5 d$ was illuminated by a $2-W$ green laser sheet ( $2 \mathrm{~mm}$ in thickness, MGL-F-532, Changchun New Industries Optoelectronics Technology Co.,
Ltd.). As suggested in Fig. 5, all of the equipment was placed on the turntable with two stainless steel stages (1 $\mathrm{m}$ diameter) and rotated together. The observed recorded images are taken in the rotating system, and the center of the cylindrical vessel and the camera axis coincided with the rotational axis of the turntable.

The turntable can rotate with a set angular velocities $\omega$ in the range of 0.0-5.0 rad/s, and the experiments used six angular velocities: $\omega=0.126,0.179,0.314,1.26,1.79$, and $3.14 \mathrm{rad} / \mathrm{s}$. As a controlling parameter, the Ekman number Ek is defined as

$$
\mathrm{Ek}=\frac{v}{2 \omega d^{2}}
$$

where $v$ is the kinematic viscosity of the fluid. With the $\omega$ values set in the present study, Ek ranged from $4.0 \times 10^{-4}$ to $1.0 \times 10^{-2}$. Further, the typical spin-up time, the Ekman timescale $t_{E}$ [28], can be estimated as

$$
t_{E}=\frac{d}{\sqrt{\nu \omega}}
$$

and distributes in the range of $11.3 \mathrm{~s} \leqslant t_{E} \leqslant 56.3 \mathrm{~s}$ here. Rotation in the counterclockwise direction was imposed on a quiescent fluid, and the camera recorded images during $60 \mathrm{~s}$ of the spin-up toward rigid-body rotation, where the fluid rotates

(a)

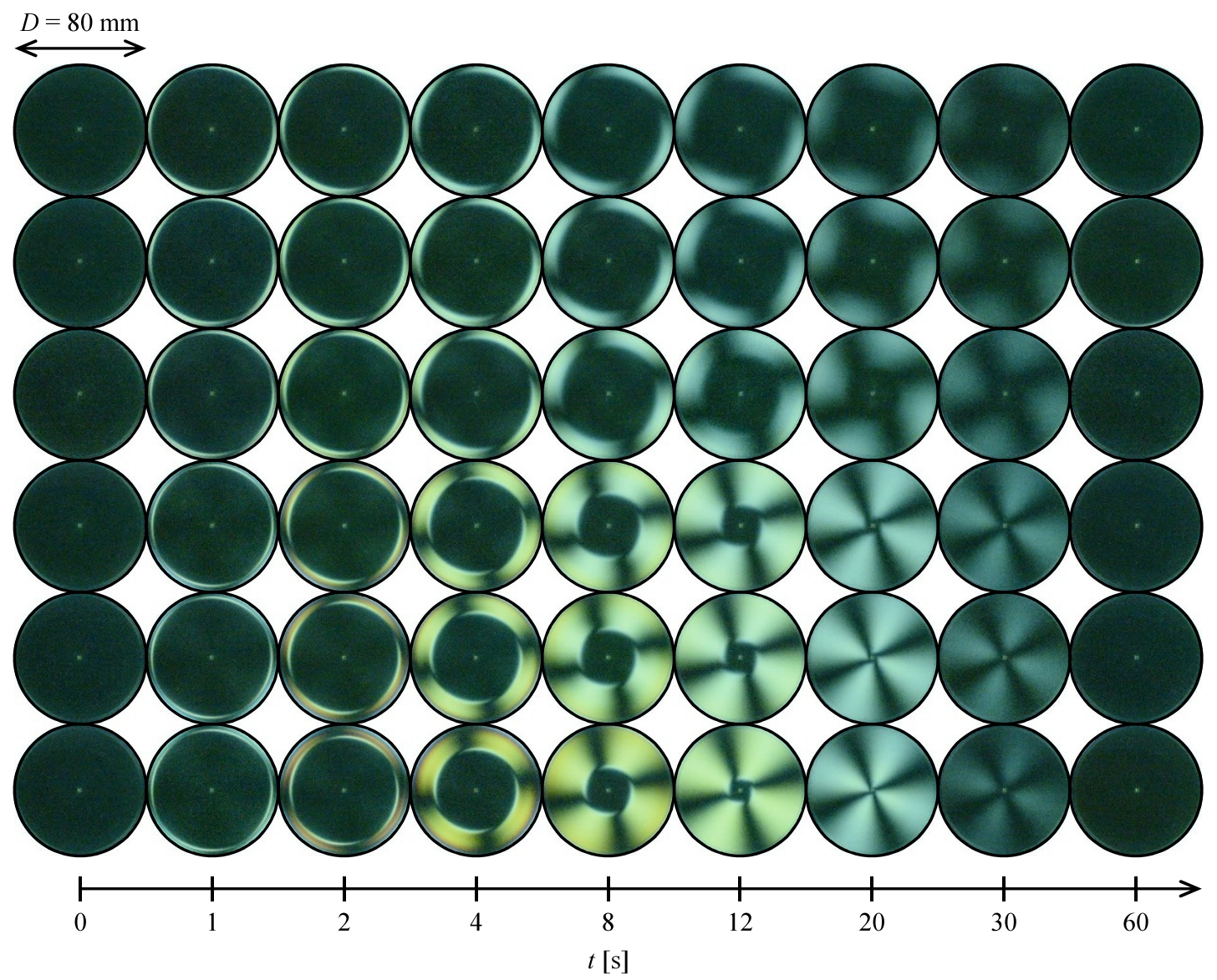

FIG. 6. Snapshots recorded as color images of the rheoscopic fluid during spin-up: (a) Ek $=1.0 \times 10^{-2}(\omega=0.126 \mathrm{rad} / \mathrm{s}),(\mathrm{b}) 7.0 \times 10^{-3}$ $(0.179 \mathrm{rad} / \mathrm{s}),(\mathrm{c}) 4.0 \times 10^{-3}(0.314 \mathrm{rad} / \mathrm{s}),(\mathrm{d}) 1.0 \times 10^{-3}(1.26 \mathrm{rad} / \mathrm{s}),(\mathrm{e}) 7.0 \times 10^{-4}(1.79 \mathrm{rad} / \mathrm{s})$, and $(\mathrm{f}) 4.0 \times 10^{-4}(3.14 \mathrm{rad} / \mathrm{s}) . \mathrm{Time}$ elapses from left to right. 
(a)

(b)
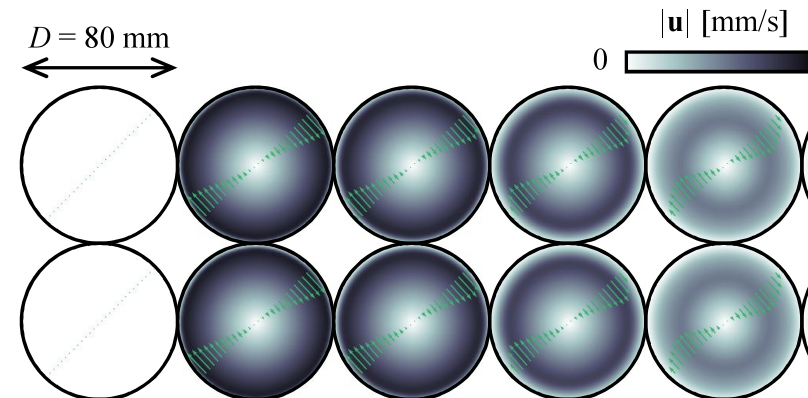

(c)

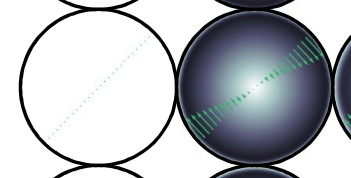

(d)

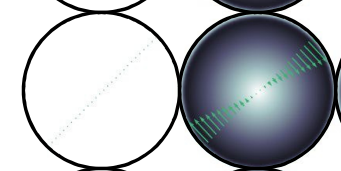

(e)
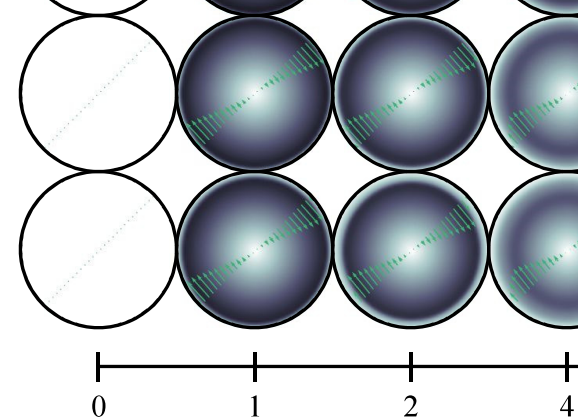

4

$t$ [s]

FIG. 7. Velocity fields at $z=0.5 d$ measured by PIV during the spin-up of water: (a) Ek $=1.0 \times 10^{-2}(\omega=0.126 \mathrm{rad} / \mathrm{s}),(\mathrm{b}) 7.0 \times 10^{-3}$ $(0.179 \mathrm{rad} / \mathrm{s}),(\mathrm{c}) 4.0 \times 10^{-3}(0.314 \mathrm{rad} / \mathrm{s}),(\mathrm{d}) 1.0 \times 10^{-3}(1.26 \mathrm{rad} / \mathrm{s}),(\mathrm{e}) 7.0 \times 10^{-4}(1.79 \mathrm{rad} / \mathrm{s}), \mathrm{and}(\mathrm{f}) 4.0 \times 10^{-4}(3.14 \mathrm{rad} / \mathrm{s}) . \mathrm{Time}$ elapses from left to right. The grayscale indicates the magnitude of the in-plane velocity $|\mathbf{u}|=\sqrt{u^{2}+v^{2}}$ obtained in the rotating frame. Velocity vectors are plotted as a radial profile with arbitrary scaling for visibility because of azimuthally symmetric flow.

together with the rotating vessel and the angular velocity becomes $v_{\theta}=r \Omega$.

\section{B. Color visualization utilizing the rheoscopic fluid}

Color snapshots recorded with the rotational system during spin-up of the rheoscopic fluid are shown in Fig. 6. The time $t=0 \mathrm{~s}$ corresponds to the rest states and are shown at the left end of each of the (a)-(f) cases in Fig. 6. At the stationary state $(t=0 \mathrm{~s})$, no color emerges in the images in any of the experiments. This is because the white light emitted from the backlight cannot penetrate the orthogonally arranged two polarization filters. This condition shows that without shear in the flow, the flake particles align randomly and there is no flow-induced birefringence. Coloration appears from the sidewall (at $t=1 \mathrm{~s}$ ) and propagates gradually toward the center of the cylinder, and multiple colors distribute with the increase in $\omega$. With time, the coloration darkens, from $t=12 \mathrm{~s}$, and at $t=60 \mathrm{~s}$ the visualized images are restored to the original dark images. In the present experimental system, the camera was rotating with the fluid container, and no fluid deformation (shear) can be observed with the camera when the flow has reached the rigid-body rotation. This is the reason why the coloration completely disappears at $t=60 \mathrm{~s}$. Considering these observed behaviors suggests the conclusion that the coloration observed in Fig. 6 originates in the flow-induced birefringence as a result of the rheoscopic fluid flows.

\section{Velocity field measurements by PIV}

Horizontal velocity fields measured by PIV are shown in Fig. 7 to compare with the color visualizations (Fig. 6). The spin-up flows are fully reproducible, and the color images in Figs. 6 and 7 can be directly compared at the same exact times. The grayscale in Fig. 7 shows the magnitude of the horizontal velocity $|\mathbf{u}|=\sqrt{u^{2}+v^{2}}$ normalized by the maximum azimuthal velocity at the sidewall $U_{\text {wall }}=D \omega / 2$, and it darkens with increases in the magnitude of the relative velocity. Stationary, at $t=0 \mathrm{~s}$ the left of the (a)-(f) panels in Fig. 7, where the figure is uniformly white, shows a zero velocity throughout the vessel. During the time when the coloration emerges in Fig. $6(t=1-30 \mathrm{~s})$, there is a momentum transfer from the sidewalls together with the development of the velocity fields. During this period, larger velocity magnitudes are shown by the greater variety in the coloration. After the flow states reach rigid-body rotation, the velocity distributions in Fig. 7 are uniformly white again, because the camera rotates together with the fluid container. Considering the direction of the velocity vectors, the flow is regarded as axisymmetric and unidirectional in the azimuthal direction 
during spin-up. Comparing Figs. 6 and 7, the coloration of the flow-induced birefringence and the flow field may be assumed to be closely related to each other. Hence we can simplify the spin-up flow as a shear-induced, axisymmetric unidirectional flow for considering the flow-induced birefringence hereafter.

\section{EVALUATION OF THE STRESS-OPTIC LAW IN NEWTONIAN FLUIDS}

For a flow during spin-up that is axisymmetric and unidirectional in the azimuthal direction, the only velocity gradient is that in the radial direction $r$ in polar coordinates $(r-\theta$ domain). This allows the principal stresses $\tau_{1}$ and $\tau_{2}$ to be disregarded, and the stress-optic law [Eq. (1)] is rewritten as

$$
\tau_{\theta}(r, t)=\frac{1}{2 C} \Delta n^{\prime}(r, t) \sin 2 \chi(r, t),
$$

according to the findings in [29,30]. In Eq. (7), $\tau_{\theta}$ is the azimuthal shear stress and $\Delta n^{\prime}$ and $\chi$ become functions of only $r$ and $t$. Further, the photoelastic coefficient $C$ is a physical property of the rheoscopic fluid and can be regarded as constant if the flake particles distribute uniformly in the fluid. Assuming that the stress-optic law [Eq. (7)] is established in the rheoscopic fluid, the combination of $\Delta n^{\prime}$ and $\chi$ must be uniquely related to $\tau_{\theta}$, and the following will attempt to explain details of the relation that ensure the establishment of the stress-optic law.

\section{A. Wave-number analysis for quantification of the orientation angle $\chi$}

The color chart (Fig. 4) involves two variables $\chi$ and $\Delta n^{\prime}$, and at least one of these two variables needs to be determined in advance to quantify the other. Considering the axisymmetry of the spin-up flow, $\chi$ would be elucidated. The dark crosses, representing isogyres [11,31], are always observed during the spin-up (see Fig. 6), and four dark regions appear along the azimuthal direction at any radial position $r$ where the fluid is deformed. The azimuthal positions of the dark regions correspond to the position where $\chi$ coincides with the optical axis of the polarizer or the analyzer (the second polarizer), i.e., the extinction angles with $\chi=m \pi / 2$. The presence of the dark crosses is consistent with Eq. (4), and thus $\chi$ can be evaluated by the inclined angle of the crosses to the $x$ and $y$ coordinates.

There is also a dark area at the center of the fluid container in Fig. 6, as there is no deformation of the fluid here; the spin-up flow does not reach this area and it remains stagnant, $\Delta n^{\prime}=0$. Expressed differently, the appearance of the isogyres at the radial position is evidence that the fluid deformation due to the spin-up propagates from the wall to the radial position. To distinguish the extinction angles $\left(\Delta n^{\prime}>0\right.$ and $\chi=$ $m \pi / 2, m \in[1,4])$ from the nondeformed regions $\left(\Delta n^{\prime}=0\right)$, the azimuthal profiles of the brightness value $Y$ would enable identification of the inclination of the dark crosses from in the system coordinate and can be simply calculated as

$$
Y=\frac{R+G+B}{3},
$$

where $R, G$, and $B$ are three brightness values of RGB (red, green, and blue) recorded for each pixel in a 24-bit format ( 8 bits for each color). An example, extracting the azimuthal
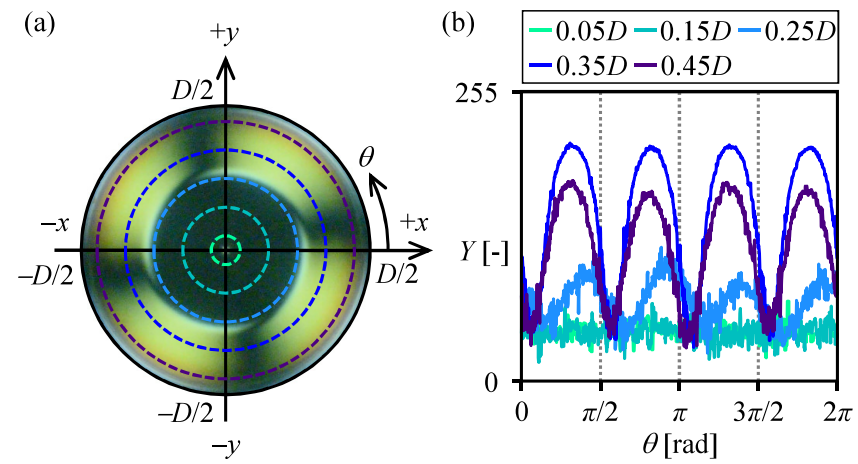

FIG. 8. Plot of azimuthal profiles of brightness $Y$ : (a) original image at $t=5 \mathrm{~s}$ in the case of $\mathrm{Ek}=4.0 \times 10^{-4}$, and (b) brightness profiles $Y$ in $\theta$ at different radial positions $(0.1,0.3,0.5,0.7$, and $0.9 D)$. Each profile corresponds to the dashed circles drawn on (a) with the same color.

profiles of $Y$ from an instantaneous image of $t=5 \mathrm{~s}$ with $\mathrm{Ek}=4.0 \times 10^{-4}$, is shown in Fig. 8(a). As indicated by the dashed lines in Fig. 8(a), $Y$ values are extracted along the $\theta$ direction at each $r$ position, and the corresponding $Y$ profiles are plotted in Fig. 8(b). The $Y$ profiles at $r \gtrsim 0.25 D$ appear in four periodic cycles $(\pi / 2)$ along the $\theta$ direction, while there is no periodicity in $Y$ at $r \leqslant 0.15 D$.

The azimuthal locations of the four peaks of the $Y$ profiles shown in Fig. 8(b) are slightly different at the different radial positions; the profile at $r=0.25 D$ is shifted towards higher angles (the $+\theta$ direction) from those at $r=0.35 D$ and $0.45 D$. This suggests that $\chi$ is a function of only $r$, i.e., it can be represented by $\chi(r)$, because the flow is regarded as axisymmetric. To determine $\chi(r)$, a discrete Fourier transform (DFT) was applied to the azimuthal profiles of $Y(r, \theta)$. An azimuthally extended brightness image of $Y(r, \theta)$ compiled from the image shown in Fig. 8(a) is shown in Fig. 9(a), and the corresponding power spectra $\widehat{Y}\left(r, k_{\theta}\right)$ are also shown in
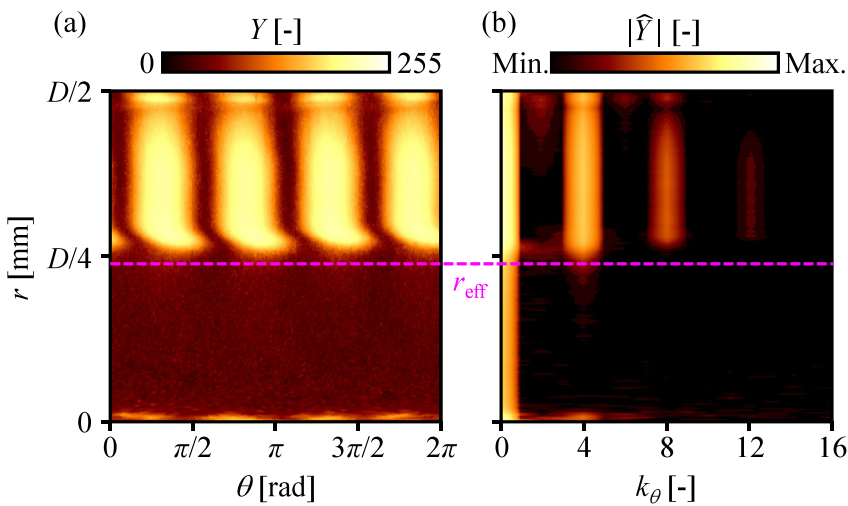

FIG. 9. (a) Pseudo-grayscale (brightness $Y$ ) image extended on $\theta$ and (b) power of brightness $|\widehat{Y}|$ in wave-number space $k_{\theta}$. Please note that the wave-number $k_{\theta}$ is shown only in a range of $0 \leqslant k_{\theta} \leqslant 16$ for visibility. The color contour of (a) is in $0 \leqslant Y \leqslant 255$, and (b) is $|\widehat{Y}|$ in logarithmic scale and normalized with the maximum and the minimum values. The displayed example corresponds to Fig. 8 $\left(\mathrm{Ek}=4.0 \times 10^{-4}\right.$ at $\left.t=5 \mathrm{~s}\right)$. Purple dashed line is the effective radius at this moment $r_{\text {eff }}=0.245 D(19.6 \mathrm{~mm})$. 
Fig. 9. The vertical axes of the graphs are the radial position $r$ and the horizontal axis of Fig. 9(a) is $\theta$, and that of Fig. 9(b) is the azimuthal wave number $k_{\theta}$. There are four brightness peaks at active regions, where the radial positions $r \gtrsim D / 4$ present fluid deformations, in Fig. 9(a). The distinct peaks in the spectra appear at the wave number of $k_{\theta}=4$, excluding the direct current component $k_{\theta}=0$ in Fig. 9(b). This makes it possible to determine the effective radius $r_{\text {eff }}$ separating the nondeformed region and the active region using the maximum peak wave number. The figure shows that if the maximum peak wave number is at $k_{\theta}=4$, the corresponding radial position is active by the shear-induced fluid flow. The minimum radial position $r$ showing a distinct wave number peak at $k_{\theta}=4$ is defined as the effective radius $r_{\text {eff }}$ dividing the nondeformed $\left(r<r_{\text {eff }}\right)$ and active regions $\left(r \geqslant r_{\text {eff }}\right)$, and is shown by the purple dashed line in Fig. 9 .

Here $Y$, corresponding to the intensity $I$, are at the maxima and minima at which $\chi=(2 m-1) \pi / 4$ and $\chi=m \pi / 2$ with arbitrary integers of $m \in[1,4]$. The brightest angle $\theta=\phi_{m}(r)$ on the image can be established by the arguments of the complex number $\widehat{Y}, \Re\left[\widehat{Y}\left(r, k_{\theta}\right)\right]$ and $\Im\left[\widehat{Y}\left(r, k_{\theta}\right)\right]$, with a fixed wave number $k_{\theta}=4$, and expressed as

$$
\phi_{m}(r)=\frac{m \pi}{2}-\frac{1}{4} \tan ^{-1}\left\{\frac{\Im\left[\widehat{Y}\left(r, k_{\theta}=4\right)\right]}{\Re\left[\widehat{Y}\left(r, k_{\theta}=4\right)\right]}\right\} .
$$

The minimum $\phi_{m}$ is placed as $\phi_{1}$ and is in the first quadrant $(0 \leqslant \theta<\pi / 2)$. The darkest angle $\phi_{m}^{\prime}(r)$, corresponding to the isogyres, can be calculated as $\phi_{m}^{\prime}(r)=\phi_{m}(r)+\pi / 4$.

The $\chi$ is the angle relative to the polarization axes of the linear polarizers ( $x$ and $y$ axes corresponding to $\theta=m \pi / 2$ ), and it is defined in a range of $0 \leqslant \chi \leqslant \pi / 4$. Its general form is written as

$$
\chi_{m}(r, \theta)=f\left[r, \Theta_{m}(r, \theta)\right],
$$

where $f$ is a function expressing the spatial distribution of $\chi$. The $\Theta_{m}$ is the angle from the darkest position $\phi_{m}^{\prime}$ and is defined as

$$
\Theta_{m}(r, \theta)=\theta-\phi_{m}^{\prime}(r)
$$

Here $\Theta_{m}$ is a cyclic value and is defined in the range of $0 \leqslant$ $\Theta_{m}<\pi / 2$. In an axisymmetric flow, the linear change of $\chi_{m}$ may be postulated along the $\theta$ directions at any $r$ position. Thus $\chi_{m}$ can be expressed as

$$
\chi_{m}(r, \theta)=\frac{\pi}{4}-\frac{1}{2} \Theta_{m}(r, \theta),
$$

and the spatial distribution of the orientation angle $\chi(r, \theta)$ is obtained.

An example determining the spatial distribution of $\chi$ is shown in Fig. 10. Figure 10(a) is the original image taken at $t=5 \mathrm{~s}$, and the processed result is presented in Fig. 10(b). In Fig. 10(b) the border of the central nondeformed and the active regions at $r=r_{\text {eff }}$ are indicated as the purple circle. The brightest positions $\phi_{m}(r)$ and the darkest positions $\phi_{m}^{\prime}(r)$ are drawn as red and blue solid lines, and the color contour shows the orientation angle $\chi(r, \theta) \in[0, \pi / 4]$. The spatial distribution of $\chi$ shown in Fig. 10(b) matches well with the brightness pattern of the original image shown in Fig. 10(a).

Incorporating all the results in this section, in the case of an axisymmetric flow, a determination of the spatial distribution
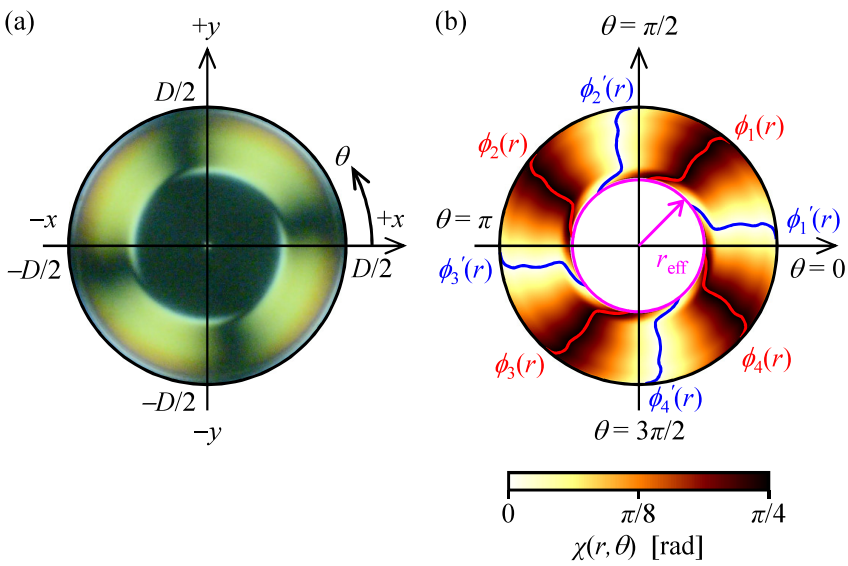

FIG. 10. An example quantifying $\chi$ with $\mathrm{Ek}=4.0 \times 10^{-4}$ at $t=5 \mathrm{~s}$ (same conditions as those of Figs. 8 and 9): (a) original color image and (b) processed result showing the effective radius $r_{\text {eff }}$ (purple circle), the brightest positions $\phi_{m}(r)$ (red lines), the darkest positions $\phi_{m}^{\prime}(r)$ (blue lines), and the orientation angle distribution $\chi(r, \theta)$ (color contours), respectively.

of $\chi$ becomes possible from a single image with satisfying both the limitation of 2D flow and requirements of temporal and spatial resolutions stated in Sec. II. That is, $\chi(r, t)$ is now accessible in Eq. (7).

\section{B. Association of color with shear stress $\tau_{\theta}$}

The flow-induced birefringence $\Delta n^{\prime}$ remains as an unknown variable in Eq. (7). To ensure the applicability if the stress-optic law, however, $\Delta n^{\prime}$ need not be obtained because $\chi$ is predetermined in Sec. IV A. At the brightest positions $\left(\theta=\phi_{m}\right)$, the reduced stress-optic law in Eq. (7) can be simplified further as

$$
\tau_{\theta}(r, t)=\frac{1}{2 C} \Delta n^{\prime}(r, t),
$$

as for $\theta=\phi_{m}$, "sin $2 \chi$ " in Eq. (7) is unity with $\chi=m \pi / 4$. In addition to the reduced stress-optic law, the transmitted light intensity in Eq. (4) can also be simplified at $\theta=\phi_{m}$ as

$$
I=I_{0} \sin ^{2} \frac{\pi \Delta n^{\prime} d}{\lambda} .
$$

Substituting Eq. (13) into Eq. (14), we obtain

$$
I=I_{0} \sin ^{2} \frac{2 \pi C d \tau_{\theta}}{\lambda} .
$$

Here $\Delta n^{\prime}$ disappears from the equations. As it is clear in Eq. (15), as long as $\chi$ is predetermined, $I$ and $\tau_{\theta}$ relate uniquely to each other, and it is possible to establish a unique relation between $I$ (or RGB values on digital images) and $\tau_{\theta}$, which is evidence ensuring that the stress-optic law holds in the rheoscopic fluid.

The coloration for $\chi=m \pi / 4$ is the most vivid, and the corresponding RGB values change a lot compared to other $\chi$ conditions as shown in Fig. 4. Figure 11 shows time-line images compiled by extracting RGB values of the brightest position $\theta=\phi_{1}(r)$ of the rheoscopic fluid visualizations (Fig. 6). The horizontal axis depicts $r$ and the vertical axis 
(a)

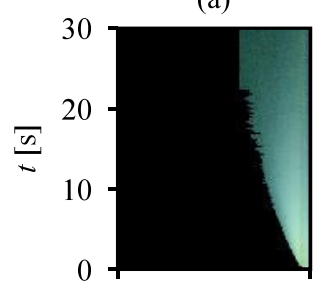

(d)

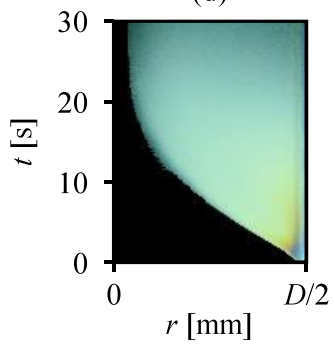

(b)

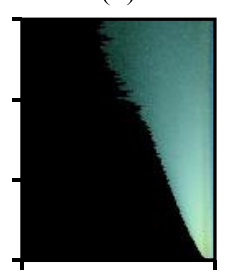

(e)
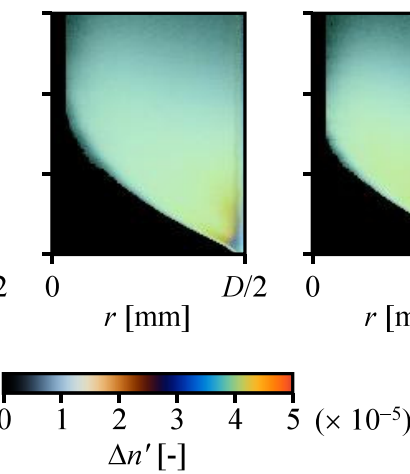

(c)

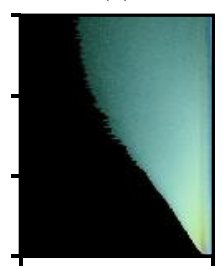

(f)

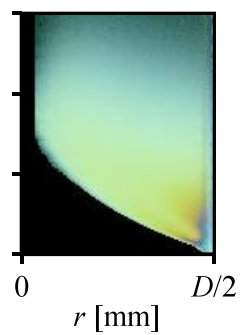

(d)

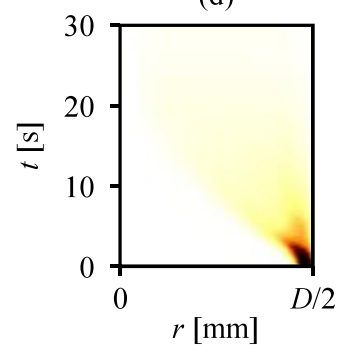

(b)
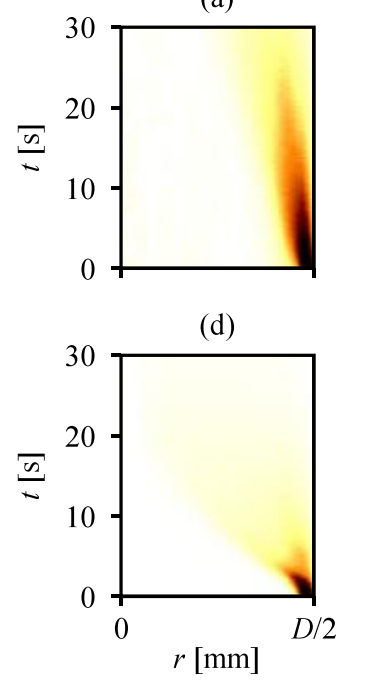

(e) (c)
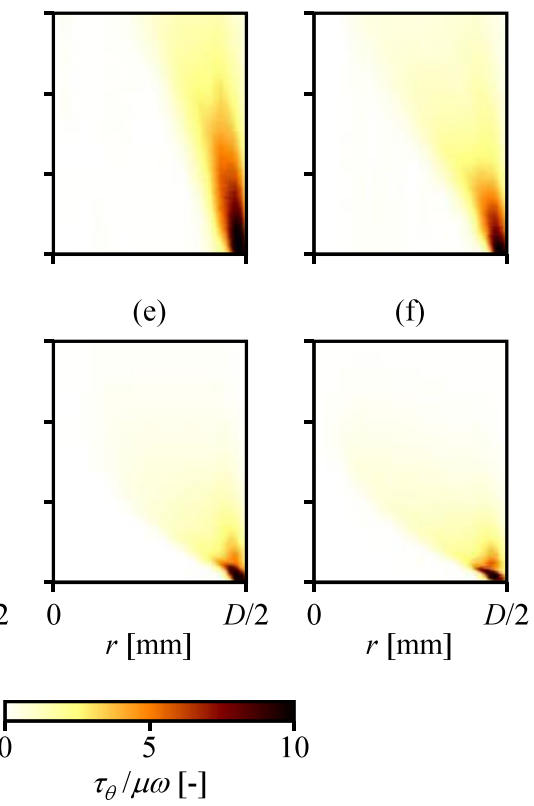

FIG. 11. Time-line images at the brightest angle $\left[\theta=\phi_{1}(r)\right]$ compiled from the snapshots (Fig. 6): (a) $\mathrm{Ek}=1.0 \times 10^{-2}$ $(\omega=0.126 \mathrm{rad} / \mathrm{s})$, (b) $7.0 \times 10^{-3}(0.179 \mathrm{rad} / \mathrm{s})$, (c) $4.0 \times 10^{-3}$ $(0.314 \mathrm{rad} / \mathrm{s}), \quad$ (d) $1.0 \times 10^{-3} \quad(1.26 \mathrm{rad} / \mathrm{s}), \quad$ (e) $7.0 \times 10^{-4}$ $(1.79 \mathrm{rad} / \mathrm{s})$, and (f) $4.0 \times 10^{-4}(3.14 \mathrm{rad} / \mathrm{s})$. Time elapses from bottom to top in $0 \leqslant t \leqslant 30 \mathrm{~s}$. The no-deformation region $\left(r<r_{\text {eff }}\right)$ appears as black. The coloration qualitatively corresponds to the birefringence of $\Delta n^{\prime}$ expressed by the color chart at $\chi=m \pi / 4$ displayed at the bottom of the panels.

shows $t$. In Fig. 11, the nondeformed regions $r<r_{\text {eff }}$ are displayed as black, and the active regions $\left(r \geqslant r_{\text {eff }}\right)$ show colors extracted from the original images. Simply, the wall-to-center progression of $r_{\text {eff }}$ may be described by the development of the sidewall viscous layer $\delta_{v} \propto \sqrt{v t}$, and $D / 2-r_{\text {eff }}$ corresponds to $\delta_{v}$. The resultant time-line images qualitatively support this, as shown by the parabolic shapes that can be seen in each panel of Fig. 11.

Using the velocity fields measured by PIV, $\tau_{\theta}$ can be estimated. The velocity vector in Cartesian coordinates $\mathbf{u}(x, y)=$ $[u, v]$ can be converted to that in the polar coordinates, $\mathbf{u}(r, \theta)=\left[v_{r}, v_{\theta}\right]$, as

$$
\left\{\begin{array}{l}
v_{r}=u \cos \theta+v \sin \theta \\
v_{\theta}=-u \sin \theta+v \cos \theta
\end{array}\right.
$$

by placing the origin at the axis of rotation (center of the cylindrical vessel). In an axisymmetric flow, $v_{r}$ is negligible compared to $v_{\theta}$, as apparent from Fig. 7, and thus $\tau_{\theta}$ is written as

$$
\tau_{\theta}(r, t)=\mu\left[\frac{\partial\left\langle v_{\theta}(r, t)\right\rangle_{\theta}}{\partial r}-\frac{\left\langle v_{\theta}(r, t)\right\rangle_{\theta}}{r}\right] .
$$

Here the azimuthal velocity $\left\langle v_{\theta}(r, t)\right\rangle_{\theta}$ is the averaged value of $v_{\theta}$ along the $\theta$ direction at a specific radius derived as

$$
\left\langle v_{\theta}(r, t)\right\rangle_{\theta}=\frac{1}{2 \pi} \int_{0}^{2 \pi} v_{\theta}(r, \theta, t) d \theta .
$$

FIG. 12. Time-line images (from bottom to top, $0 \leqslant t \leqslant 30 \mathrm{~s}$ ) of the azimuthal shear stress $\tau_{\theta}$ : (a) $\mathrm{Ek}=1.0 \times 10^{-2}$ $(\omega=0.126 \mathrm{rad} / \mathrm{s})$, (b) $7.0 \times 10^{-3}(0.179 \mathrm{rad} / \mathrm{s})$, (c) $4.0 \times 10^{-3}$ $(0.314 \mathrm{rad} / \mathrm{s}), \quad$ (d) $1.0 \times 10^{-3} \quad(1.26 \mathrm{rad} / \mathrm{s}), \quad$ (e) $7.0 \times 10^{-4}$ $(1.79 \mathrm{rad} / \mathrm{s})$, and (f) $4.0 \times 10^{-4}(3.14 \mathrm{rad} / \mathrm{s})$. Color contours of shear stress $\tau_{\theta}$ are scaled by $\mu \omega$.

This manipulation using the azimuthal average is possible in an axisymmetric flow and minimizes measurement noise in the calculation of the derivative in Eq. (17). The estimated shear stress fields extended on the time axes are shown in Fig. 12. Here, $\tau_{\theta}$ of each case are scaled by $\mu \omega$ to enhance visibility. Commonly, intense shear stress regions indicated as dark colors gradually propagate to the center of the fluid container. The faster rotation (smaller Ek) cases show faster propagation of the shear stress. This is apparent in the Ekman timescale $t_{E}$, which is proportional to $\omega^{-1 / 2}$, and is easily understood from the development of the sidewall viscous layer as $\delta_{v} \sim \sqrt{v t}$. The active regions shown in Fig. 11 correspond well to the high- $\tau_{\theta}$ regions in Fig. 12.

To compare the recorded color information with the theoretical color chart shown in Fig. 4, the colors in Fig. 11(f) are projected into the $\alpha-\beta$ domain (the polar chromaticity coordinate), as shown in Fig. 13. Here the $\alpha-\beta$ domain is a color expression method obtained from RGB values by projecting the RGB Cartesian cube to a 2D domain of $\alpha-\beta$, and $\alpha$ and $\beta$ are respectively derived as

$$
\alpha=\frac{1}{2}(2 R-G-B)
$$

and

$$
\beta=\frac{\sqrt{3}}{2}(G-B)
$$

In Fig. 13, the scatter plot is colored with the actually recorded colors shown in Fig. 11(f). Distribution of the plotted data forms a stretched elliptical shape rising to the right, regardless of brightness or vividness. The solid line, swirling from 


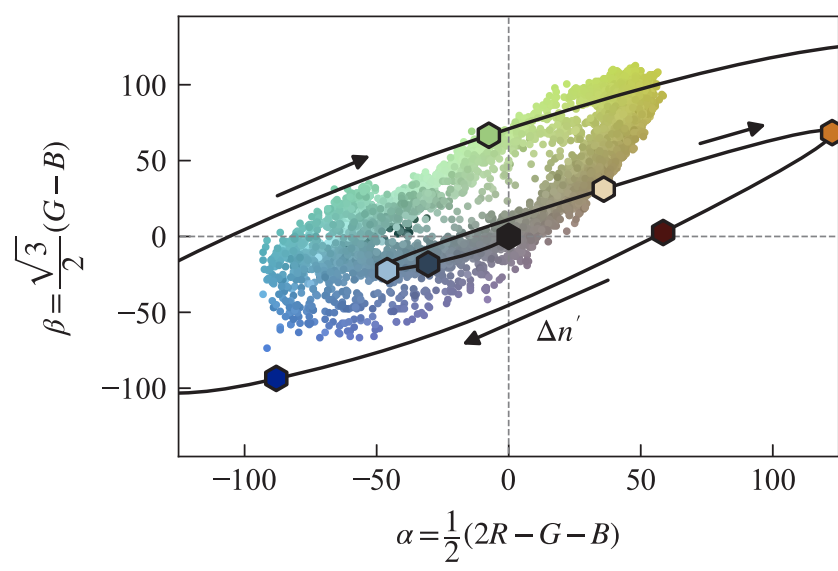

FIG. 13. Plot of the recorded and the theoretical color values compared in the $\alpha-\beta$ domain. Solid line shows the theoretical values accessible from the color chart in a range from $\Delta n^{\prime}=0$ to $5.0 \times 10^{-5}$. Hexagonal symbols are plotted every $5.0 \times 10^{-6}$ from $\Delta n^{\prime}=0$ on the line and colored in the same color to the color chart.

$(\alpha, \beta)=(0,0)$ outward with increases in $\Delta n^{\prime}$, shows the theoretical values accessible from the color chart in Fig. 4(b). The hexagonal symbols are plotted at every $5.0 \times 10^{-6}$ from $\Delta n^{\prime}=0$ and colored in the same color to the color chart. The elliptical distributions are similar to each other, while there is a considerable discrepancy which may originate in the nonideal optical environment. However, it is possible to qualitatively consider the coloration of the rheoscopic fluid as the interference color from the plot in Fig. 13.

The spin-up is fully reproducible, and the spatial and temporal distributions of the RGB values in Fig. 11 and $\tau_{\theta}$ in Fig. 12 can be compared. The correspondence between the $\tau_{\theta}$ and color values with the case of Ek $=4.0 \times 10^{-4}$ is shown in Fig. 14 as the RGB domain of (a), the $R B$ domain of (b), the $B G$ domain of (c), and the $R G$ domain of (d). Here the timeline images of the four brightest angles $\left(\theta=\phi_{1}, \phi_{2}, \phi_{3}\right.$, and $\left.\phi_{4}\right)$ are averaged to reduce spiky noises originating from the ISO sensitivity of the camera. The spatiotemporal distribution of $\tau_{\theta}$ is interpolated in the $r$ direction using the third-order spline interpolation to fit the number of data points of $\tau_{\theta}$ with that of the pixel RGB values. Conventionally, the color information is expressed as projected values such as $\alpha-\beta$ or hue (the angular component in the $\alpha-\beta$ domain) to simplify the manipulations of the color information, reducing the original information. In Figs. 13 and 14(b)-14(d), however, the unique relation between $\tau_{\theta}$ and the color values cannot be simply discriminated in any of the $2 \mathrm{D}$ color domains. In the threedimensional domain of RGB shown in Fig. 14(a), $\tau_{\theta}$ can be identified uniquely, even though the deviations in the color increase with the increase in the $\tau_{\theta}$. The deviations might be unique in the present spin-up experiment, which induced highshear-stress regions at the sidewall corresponding to the edge of the visualized images. Overall, $\tau_{\theta}$ is successfully associated with the RGB values without reducing the color information, even with such deviations. In conclusion, it is verified that the stress-optic law can be considered to be established in the rheoscopic fluid, as Eq. (15) is shown to hold here.
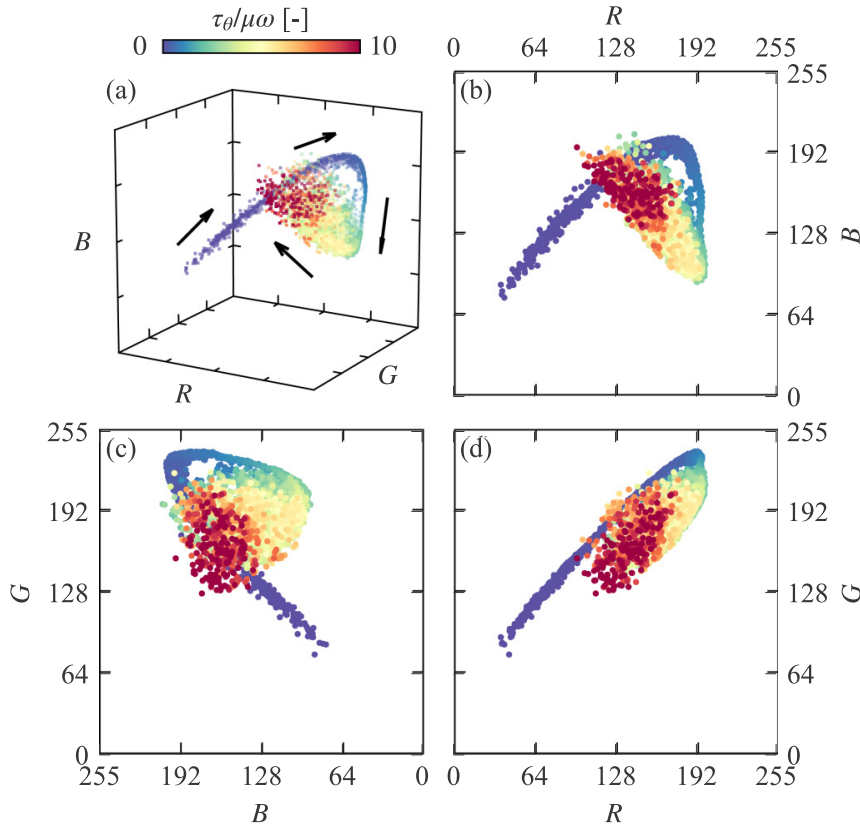

FIG. 14. The $\tau_{\theta}$ and RGB values plotted in different domains: (a) RGB domain, (b) RB domain, (c) BG domain, and (d) RG domain. Plots are colored with $\tau_{\theta}$ normalized by $\mu \omega$. Black arrows in (a) guide the increase in $\tau_{\theta}$.

As the validity of the stress-optic law in the rheoscopic fluid is established, the magnitude of the photoelastic coefficient $|C|$ of the rheoscopic fluid can be estimated by using representative values of $\Delta n^{\prime}$ and $\tau_{\theta}$. In Fig. 11(f), the maximum value of $\Delta n^{\prime}$ is approximated as $\Delta n_{\max }^{\prime} \sim 4 \times 10^{-5}$ from the coloration. Similarly, the maximum value of $\tau_{\theta}$ takes $\tau_{\theta \max } \sim 5 \times 10^{-3} \mathrm{~Pa}$ in Fig. 12(f). Using Eq. (13), $|C|$ is estimated as $4 \times 10^{-3}=O\left(10^{-3} \mathrm{~Pa}^{-1}\right)$. In comparison, the order of $|C|$ was measured as $O\left(10^{-7} \mathrm{~Pa}^{-1}\right)$ in wormlike micellar solutions $[29,30]$. For the solid materials, the $|C|$ values are frequently expressed in the unit of Brewster $\left(=10^{-12} \mathrm{~Pa}^{-1}\right)$. Considering these, the flow-induced birefringence in the rheoscopic fluid is very sensitive to stress and may display colors even with a weak stress field imposed.

\section{SUMMARY AND OUTLOOK}

To establish the stress-optic law as being held in Newtonian fluids, we investigated a rheoscopic fluid showing the flowinduced birefringence in a direct stress field measurement of fluid flows. Two types of spin-up experiments ensuring 2D flows were conducted to fulfill the requirements of high temporal and spatial resolutions for the photoelastic analysis in fluid flows. One experiment was a color visualization using the rheoscopic fluid, and another was velocity field measurements by PIV using water. The wave-number analysis allowed a successful quantification of the spatial distribution of the orientation angle $\chi$ from an instantaneous color image by the results of the axisymmetric flow measurements. Subsequent direct comparisons of the RGB values from the color visualization and the $\tau_{\theta}$ estimated from the velocity fields measured by PIV elucidated a unique relation between these 
two parameters, verifying the validity of the stress-optic law in the fluid.

Detailed discussion of the quantification method of the stress field is not attempted here, because the aim of the present study set out to ensure the validity of the stress-optic law with the rheoscopic fluid. To utilize the stress-optic law in a straightforward way, $\Delta n^{\prime}$ is required to be determined in addition to $\chi$. There have been reports of a determination of $\Delta n^{\prime}$ elsewhere (e.g., [7,8,32]). To fulfill the requirements mentioned in Sec. II D, the so-called RGB photoelasticity (e.g., [32-34]) and the colorimetry-based retardation measurement method (e.g., [35]) would seem to offer plausible solutions because they use a single color camera and relatively simple optical settings. These methods measure $\Delta n^{\prime}$ or $\Gamma$ from the color information, and the stress-optic law can be utilized if the photoelastic coefficient $C$ is predetermined. As an alternative, direct association between stress values and colors would offer a plausible way to quantify the stress fields by performing a reference (calibration) experiment, because $\Delta n^{\prime}$ (or $C$ ) is not necessarily required in practice as shown by Eq. (15).
The present case successfully identified $\tau_{\theta}$ by using all the RGB values in the $3 \mathrm{D}$ domain. To solve this kind of multiple input regression problems, an artificial neural network (ANN) would be a practical solution. The application of ANN to a similar color fluid measurement as the one reported here can be seen in liquid crystal thermometry (e.g., [36]). Although the applicable range of the stress field measurements needs more consideration in terms of the flow intensity, incorporating these various methods, temporally and spatially resolved stress field measurements in Newtonian fluids utilizing the stress-optic law, would become possible in future research.

\section{ACKNOWLEDGMENTS}

We wish to express our gratitude to Dr. S. Nomura for recording the microscope photos shown in Fig. 2. This work was supported by Grant-in-Aids for JSPS Fellows (Grant No. JP19J20096) and Scientific Research B (Grant No. JP19H02057).
[1] R. J. Adrian, Twenty years of particle image velocimetry, Exp. Fluids 39, 159 (2005).

[2] M. Raffel, C. E. Willert, F. Scarano, C. J. Kähler, S. T. Wereley, and J. Kompenhans, Particle Image Velocimetry: A Practical Guide (Springer, New York, 2018).

[3] C. J. Kähler, T. Astarita, P. P. Vlachos, J. Sakakibara, R. Hain, S. Discetti, R. La Foy, and C. Cierpka, Main results of the 4th International PIV Challenge, Exp. Fluids 57, 97 (2016).

[4] C. Tropea and A. L. Yarin, Springer Handbook of Experimental Fluid Mechanics (Springer Science \& Business Media, New York, 2007).

[5] S. Hosokawa, T. Fukunaga, and A. Tomiyama, Application of photobleaching molecular tagging velocimetry to turbulent bubbly flow in a square duct, Exp. Fluids 47, 745 (2009).

[6] S. Hosokawa, T. Suzuki, and A. Tomiyama, Turbulence kinetic energy budget in bubbly flows in a vertical duct, Exp. Fluids 52, 719 (2012).

[7] E. A. Patterson, Digital photoelasticity: Principles, practice and potential, measurements lecture, Strain 38, 27 (2002).

[8] K. Ramesh, T. Kasimayan, and B. Neethi Simon, Digital photoelasticity-A comprehensive review, J. Strain Anal. Eng. Des. 46, 245 (2011).

[9] W. Philippoff, Flow birefringence and stress, J. Appl. Phys. 27, 984 (1956).

[10] A. Peterlin, Optical effects in flow, Annu. Rev. Fluid Mech. 8, 35 (1976).

[11] D. S. Pearson, A. D. Kiss, L. J. Fetters, and M. Doi, Flowinduced birefringence of concentrated polyisoprene solutions, J. Rheol. 33, 517 (1989).

[12] P. Matisse and M. Gorman, Neutrally buoyant anisotropic particles for flow visualization, Phys. Fluids 27, 759 (1984).

[13] N. Abcha, N. Latrache, F. Dumouchel, and I. Mutabazi, Qualitative relation between reflected light intensity by Kalliroscope flakes and velocity field in the Couette-Taylor flow system, Exp. Fluids 45, 85 (2008).
[14] S. Goto, S. Kida, and S. Fujiwara, Flow visualization using reflective flakes, J. Fluid Mech. 683, 417 (2011).

[15] M. Byron, J. Einarsson, K. Gustavsson, G. Voth, B. Mehlig, and E. Variano, Shape-dependence of particle rotation in isotropic turbulence, Phys. Fluids 27, 035101 (2015).

[16] D. Borrero-Echeverry, C. J. Crowley, and T. P. Riddick, Rheoscopic fluids in a post-Kalliroscope world, Phys. Fluids 30, 087103 (2018).

[17] D. L. Hu, L. Mendel, B. Chan, T. Goreau, and J. W. M. Bush, Visualization of a fish wake using tobacco mosaic virus, Phys. Fluids 17, 091103 (2005).

[18] D. L. Hu, T. J. Goreau, and J. W. M. Bush, Flow visualization using tobacco mosaic virus, Exp. Fluids 46, 477 (2009).

[19] Laminar Science Corporation, http://www.laminarsciences. com/, accessed: 2020.

[20] R. Blackstock, US Patent 7,210,809. (2007)

[21] R. K. Blackstock, US Patent 7,334,910 (2008).

[22] B. E. Sørensen, A revised Michel-Lévy interference color chart based on first-principles calculations, Eur. J. Mineral. 25, 5 (2012).

[23] T. Onuma and Y. Otani, A development of two-dimensional birefringence distribution measurement system with a sampling rate of 1.3 MHz, Opt. Commun. 315, 69 (2014).

[24] E. H. Wedemeyer, The unsteady flow within a spinning cylinder, J. Fluid Mech. 20, 383 (1964).

[25] H. P. Greenspan, The Theory of Rotating Fluids (Cambridge University Press, Cambridge, England, 1968).

[26] E. R. Benton and A. Clark Jr., Spin-up, Annu. Rev. Fluid Mech. 6, 257 (1974).

[27] P. D. Weidman, On the spin-up and spin-down of a rotating fluid. Part 1. Extending the Wedemeyer model, J. Fluid Mech. 77, 685 (1976).

[28] H. P. Greenspan and L. N. Howard, On a time-dependent motion of a rotating fluid, J. Fluid Mech. 17, 385 (1963). 
[29] T. Shikata, S. J. Dahman, and D. S. Pearson, Rheooptical behavior of wormlike micelles, Langmuir 10, 3470 (1994).

[30] M. Ito, Y. Yoshitake, and T. Takahashi, Shear-induced structure change in shear-banding of a wormlike micellar solution in concentric cylinder flow, J. Rheol. 60, 1019 (2016).

[31] Y.-K. Kim, B. Senyuk, and O. D. Lavrentovich, Molecular reorientation of a nematic liquid crystal by thermal expansion, Nat. Commun. 3, 1133 (2012).

[32] A. Ajovalasit, G. Petrucci, and M. Scafidi, Review of RGB photoelasticity, Opt. Lasers Eng. 68, 58 (2015).
[33] A. Ajovalasit, S. Barone, and G. Petrucci, Towards RGB photoelasticity: Full-field automated photoelasticity in white light, Exp. Mech. 35, 193 (1995).

[34] A. Ajovalasit, G. Petrucci, and M. Scafidi, RGB photoelasticity: Review and improvements, Strain 46, 137 (2010).

[35] C. C. Montarou, T. K. Gaylord, R. A. Villalaz, and E. N. Glytsis, Colorimetry-based retardation measurement method with white-light interference, Appl. Opt. 41, 5290 (2002).

[36] S. Anders, D. Noto, Y. Tasaka, and S. Eckert, Simultaneous optical measurement of temperature and velocity fields in solidifying liquids, Exp. Fluids 61, 113 (2020). 